\title{
Gene expression and plant hormone levels in two contrasting rice genotypes responding to brown planthopper infestation
}

Changyan Li', Chao Luo', Zaihui Zhou', Rui Wang ${ }^{1}$, Fei Ling ${ }^{1}$, Langtao Xiao², Yongjun Lin ${ }^{1}$ and Hao Chen ${ }^{1 *}$ (D)

\begin{abstract}
Background: The brown planthopper (BPH; Nilaparvata lugens Stål) is a destructive piercing-sucking insect pest of rice. The plant hormones salicylic acid (SA) and jasmonic acid (JA) play important roles in plant-pest interactions. Many isolated rice genes that modulate $\mathrm{BPH}$ resistance are involved in the metabolism or signaling pathways of SA, $\mathrm{JA}$ and ethylene. 'Rathu Heenati' (RH) is a rice cultivar with a high-level, broad-spectrum resistance to all BPH biotypes. Here, RH was used as the research material, while a BPH-susceptible rice cultivar 'Taichung Native 1' (TN1) was the control. A cDNA microarray analysis illuminated the resistance response at the genome level of RH under BPH infestation. The levels of SA and JA in RH and TN1 seedlings after BPH infestation were also determined.

Results: The expression pattern clustering indicated that 1467 differential probe sets may be associated with constitutive resistance and 67 with the $\mathrm{BPH}$ infestation-responsive resistance of $\mathrm{RH}$. A Venn diagram analysis revealed $192 \mathrm{RH}$-specific and BPH-inducible probe sets. Finally, 23 BPH resistance-related gene candidates were selected based on the expression pattern clustering and Venn diagram analysis. In $\mathrm{RH}$, the SA content significantly increased and the JA content significantly decreased after BPH infestation, with the former occurring prior to the latter. In $\mathrm{RH}$, the differential genes in the SA pathway were synthesis-related and were up-regulated after BPH infestation. The differential genes in the JA pathway were also up-regulated. They were jasmonate ZIM-domain transcription factors, which are important negative regulators of the JA pathway. Comparatively, genes involved in the ET pathway were less affected by a BPH infestation in RH. DNA sequence analysis revealed that most BPH infestation-inducible genes may be regulated by the genetic background in a trans-acting manner, instead of by their promoters.
\end{abstract}

Conclusions: We profiled the analysis of the global gene expression in RH and TN1 under BPH infestation, together with changes in the SA and JA levels. SA plays a leading role in the resistance response of rice to $\mathrm{BPH}$. Our results will aid in understanding the molecular basis of RH's BPH resistance and facilitate the identification of new resistance-related genes for breeding BPH-resistant rice varieties.

Keywords: Oryza sativa, Nilaparvata lugens, cDNA microarray, Insect-resistance

\footnotetext{
* Correspondence: hchen@mail.hzau.edu.cn

${ }^{1}$ National Key Laboratory of Crop Genetic Improvement and National Centre

of Plant Gene Research (Wuhan), Huazhong Agricultural University, Wuhan

430070, China

Full list of author information is available at the end of the article
} 


\section{Background}

As a staple food for more than half of the world's population, rice (Oryza sativa) is the most important crop in Asia [1], and insect pests are a main factor affecting rice production. Rice brown planthopper (BPH; Nilaparvata lugens Stål), which sucks sap from the plant's phloem, is highly destructive [2]. In addition to direct damage, $\mathrm{BPH}$, as the intermediate vector, can infect rice with pathogens, such as grass stunt virus and ragged stunt virus, leading to further yield losses [3, 4].

Since the 1960s, rice breeders have endeavored to identify BPH-resistant germplasms and develop BPH-resistant rice varieties. At least $30 \mathrm{BPH}$-resistance quantitative trait loci (QTLs) have been identified in rice. Among these, the chromosomal locations of 21 have been determined, and 12 have been fine-mapped. Most of these BPH-resistance genes were identified from wild rice, few from indica rice and none from japonica rice [5-7].

Four major BPH-resistance QTLs, Bph14, Bph3/ Bph17, BPH26/BPH2 and Bph29, were cloned using map-based techniques [8-11]. Bph14 and BPH26 encode coiled-coil, nucleotide-binding and leucine-rich repeat proteins that resemble the $R$ genes of the nucleotidebinding-leucine-rich repeat family, which mediate plant resistance to pathogens $[8,10]$. Bph14 and BPH26 are presumed to induce effector-triggered immunity, like the $R$ genes, because they share similar conserved protein domains. The expression of Bph14 is induced by $\mathrm{BPH}$ infestation, and it then activates the salicylic acid (SA) signaling pathway, induces calluses deposition in phloem cells and enhances trypsin inhibitor production [8]. $B P H 26$, like Bph14, also mediates sucking inhibition in the phloem sieve element. The Bph3 locus is a cluster of three genes encoding plasma membrane-localized lectin receptor kinases (OsLecRK1-OsLecRK3). Bph3 was proposed to play a critical role in priming the patterntriggered immunity response to $\mathrm{BPH}$ infestation by perceiving herbivore-associated or damage-associated molecular patterns due to the functions of recently discovered lectin receptor kinases [9]. Bph29 is a recessive resistance gene that encodes a B3 DNA-binding domain that contains a protein that has a DNA mutation in the B3 domain. Bph29 was proposed to have lost the function of the dominant allele, which is required for the settling of insects, and thus confers an antixenosis resistance in conjunction with an anther recessive locus [11]. The cloning of major BPH-resistance QTLs facilitated the understanding of the molecular mechanisms of resistant rice to $\mathrm{BPH}$, although the biological mechanisms are not well understood.

In addition to the major BPH-resistance QTLs, many other rice genes that modulate $\mathrm{BPH}$ resistance (resistance-related genes), such as OsHI-LOX [12], Bphi008a [13], OsERF3 [14], OsPLD $\alpha 4$ and $\alpha 5$ [15], OsHPL3 [16],
OsACS2 [17], OsAOC [18], Osr9-LOX1 [19], and OsJMT1 [20], have recently been isolated. Most of them were identified using a reverse genetics strategy, and all of them are involved in the metabolism or signaling pathways of the plant hormones SA, jasmonic acid (JA) and ethylene (ET), indicating that these hormones play important roles in rice defense responses to $\mathrm{BPH}$. SA is generally considered to positively regulate plant defense responses against pathogens and piercing-sucking insects, which cause minimal levels of physical injury to the host plants. The major BPH-resistance QTLs Bph14 and $\mathrm{BPH} 29$ enhance the expression of the SA synthesisrelated genes and the homolog of Arabidopsis nonexpressor of pathogenesis-related genes 1 , a key regulator of SA-dependent systemic acquired resistance after $\mathrm{BPH}$ infestation. They also suppress the expression levels of the JA synthesis-related genes and the ethylene signaling pathway receptor gene ethylene insensitive $2[8,11]$.

JA is associated with wounding responses and positively regulates the plant defense response against chewing insects that cause extensive damage to the host. The interactions between SA and JA are commonly antagonistic, with SA having a suppression effect on JA accumulation and signaling. ET is considered to finetune the JA-induced responses, and JA and ET are synergistic. Many rice genes involved in JA metabolism, including OsHI-LOX [12], OsPLDa4 and -a5 [13], OsHPL3 [16], AOC [18], Osr9-LOX1 [19] and OsJMT1 [20], can modulate $\mathrm{BPH}$ resistance. JA and its metabolites have diverse functions in $\mathrm{BPH}$ resistance. The ET pathway is generally considered to negatively modulate the $\mathrm{BPH}$ resistance of rice [21]. The ET-responsive gene OsERF3 encodes a nucleus-localized protein, which is rapidly up-regulated in response to infestations of the rice striped stem borer (SSB; Chilo suppressalis Walker). Transgenic rice overexpressing OsERF3 exhibit improved SSB resistance but are more susceptible to $\mathrm{BPH}$. OsACS2 encodes a rice 1-aminocyclopropane-1-carboxylic acid synthase gene, which is inducible by both SSB and $\mathrm{BPH}$ infestations. Suppression of OsACS2 expression in rice reduced the elicited ethylene emissions and SSB resistance, but enhanced $\mathrm{BPH}$ resistance [17].

'Rathu Heenati' (RH), an indica rice cultivar from Sri Lanka, has a high, durable resistance to all of the BPH biotypes. $\mathrm{RH}$ is regularly used as a positive control when identifying $\mathrm{BPH}$ resistant rice varieties and is an important $\mathrm{BPH}$-resistance donor for rice breeding. In this study, a cDNA microarray analysis was performed to profile the whole genome expression of $\mathrm{RH}$, and a $\mathrm{BPH}$ susceptible rice cultivar 'Taichung Native 1' (TN1) was used as the control. All of the rice plants were grown under three treatment conditions, natural growth (untreated), needle puncturing (mocking a simple mechanical wound) and $\mathrm{BPH}$ infestation, and two sampling time 
points, 6 and $24 \mathrm{~h}$ after treatment, were used. The changes in the levels of SA and JA in RH and TN1 seedlings were also determined at 6,24 and $48 \mathrm{~h}$ after $\mathrm{BPH}$ infestation. The ET content is difficult to measure because of its volatility and was, therefore, not determined in this study. This study revealed the resistance responses of RH to $\mathrm{BPH}$ at the genomic transcription level and through changes in the JA and SA contents. The results of this study aid in understanding the biological foundation of the BPH resistance of $\mathrm{RH}$ and in identifying new $\mathrm{BPH}$ resistance-related genes for rice breeding.

\section{Results}

\section{Genome-wide comparison of differentially-expressed} probe sets between RH and TN1 under BPH infestation

The experimental design included two rice varieties $(\mathrm{RH}$ and TN1), three treatments (untreated control, needle puncturing and $\mathrm{BPH}$ infestation) and two sampling time points (6 and $24 \mathrm{~h}$ after $\mathrm{BPH}$ infestation). There were 12 samples in total, with 3 biological replicates per sample. The abbreviations R6C, R24C, R6N, R24N, R6P, R24P, T6C, T24C, T6N, T24N, T6P and T24P were used to represent these 12 samples, where the first letter, $\mathrm{R}$ or $\mathrm{T}$, indicates the rice varieties $\mathrm{RH}$ or $\mathrm{TN} 1$, respectively; the
Arabic number in the middle indicates the 6 or $24 \mathrm{~h}$ post-treatment collection time points; and the last letter $\mathrm{C}, \mathrm{N}$ or $\mathrm{P}$, indicates one of the three different treatments, untreated control, needle puncturing or $\mathrm{BPH}$ infestation, respectively. The Affymetrix GeneChip Rice Genome Array, containing 57,194 probe sets, was used for the microarray analysis, and the results of all 36 chips were submitted to the NCBI website (http:// www.ncbi.nlm.nih.gov/geo/; accession number: GSE74 106; Additional file 1).

There were 8019 differentially-expressed probe sets [fold change $(\mathrm{FC}) \geq 2$ ] detected in this study. These included 3682 differential probe sets between different treatments, 3339 between different varieties ( $\mathrm{RH}$ and TN1), and 5757 between different sampling times, with the two non-differentiating variables being the same (Additional file 2). To compare the gene expressional differences between RH and TN1, all 8019 differential probe sets were clustered into different groups based on their expression patterns using Multi-Experiment Viewer (Fig. 1). Significantly, 785 differential probe sets in Group k had low expression levels in TN1 and high expression levels in RH under all of the conditions; in contrast, 682 differential probe sets in Group p had low

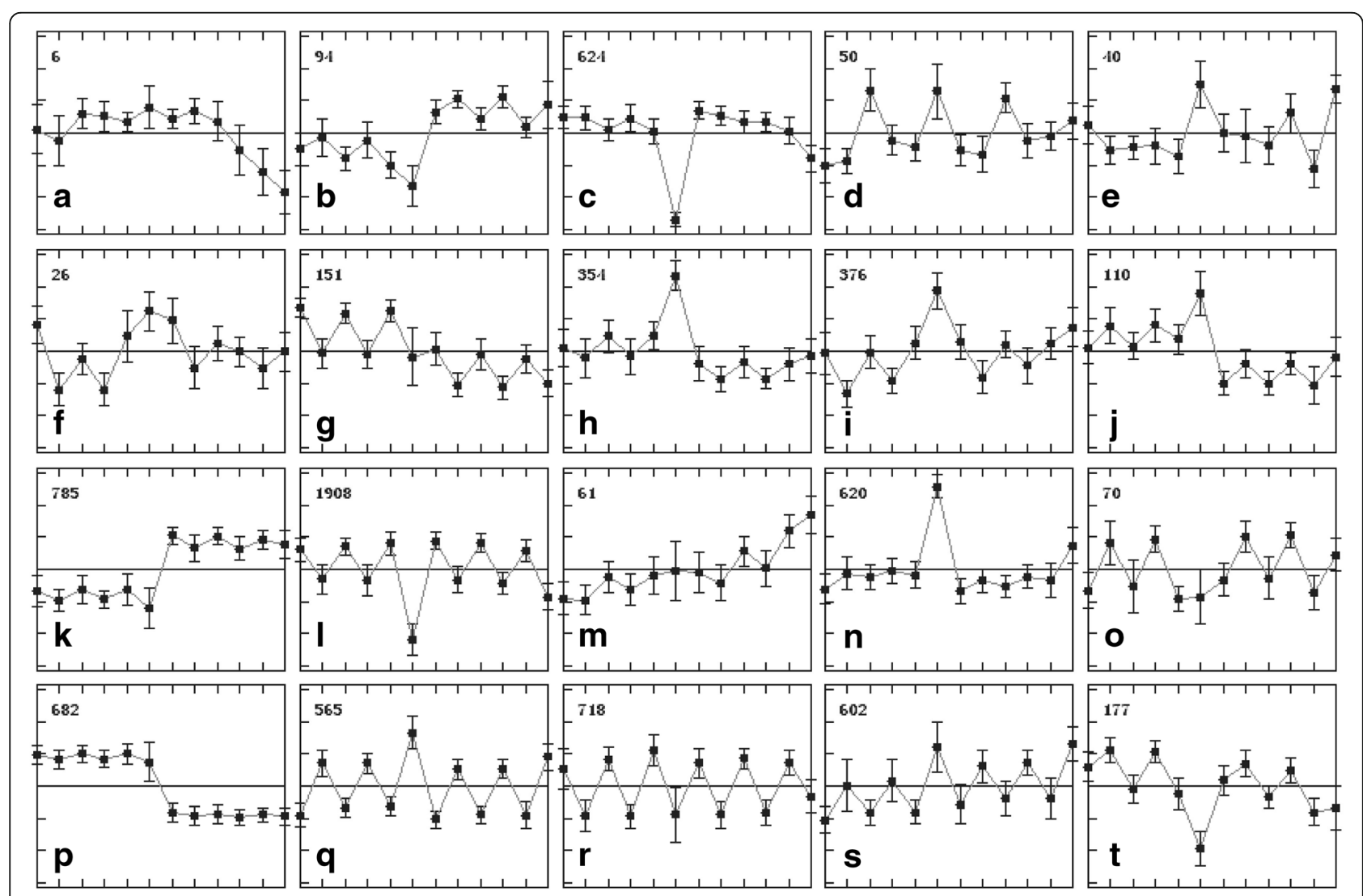

Fig. 1 Expression pattern clustering of the differential genes in 12 samples. The points in each panel are from left-to-right: T6C, T24C, T6N, T24N, T6P, T24P, R6C, R24C, R6N, R24N, R6P and R24. a-t present 20 types of expression patterns of the differential genes that are classified by the software Multi Experiment Viewer 
expression levels in $\mathrm{RH}$ and high expression levels in TN1 under all of the conditions. Therefore, Groups k and $\mathrm{p}$ roughly represent the genetic background differences between RH and TN1. Genes related to the constitutive resistance of $\mathrm{RH}$ should be included in these two groups. Groups a and $\mathrm{m}$ represent probe sets with $\mathrm{BPH}$ infestation-specific down- (6 differential probe sets) and up-regulated (61 differential probe sets) expression levels in $\mathrm{RH}$, respectively. Genes in these two probe sets may be related to the $\mathrm{BPH}$ infestation-responsive resistance of RH. Differential probe sets in Groups l, q, r and s showed regular fluctuations associated with sampling time, indicating that their expression is mainly controlled by sampling time (circadian rhythm or other environmental factors). These four types of expression patterns included a total of 3793 differential probe sets, accounting for almost a half of the 8019 differential probe sets.

\section{Best reference genes for $\mathrm{BPH}$ resistance research}

Reference genes are key factors that ensure the accuracy of a gene expressional analysis under specific conditions. It was very important to select a stably expressed gene under $\mathrm{BPH}$ infestation as the reference for quantitative reverse transcription polymerase chain reaction (qRTPCR) analysis [22-24]. Figure 2 shows eight frequentlyused reference genes, actin-1 (Os03g50890), LSD1 (Os12 g41700), GAPDH (Os02g38920), SDHA (Os07g04240), TPB (Os02g45410), RPS27a (OsO1g22490), HSP (OsO3g 31300 ) and Ubiquitin (Os03g03920), and their respective signal values extracted from our microarray data under different treatments. Actin-1 and RPS27 $\alpha$ were downregulated in both $\mathrm{TN} 1$ and $\mathrm{RH}$ within $24 \mathrm{~h}$ after $\mathrm{BPH}$ infestation; GAPDH showed an obvious expressional difference between RH and TN1; and the expression level of LSD1 was stable but relatively low. Among the remaining four references, $T B P$ and Ubiquitin showed better overall consistency compared with $H S P$ and $S D H A$. Therefore, TBP and Ubiquitin were determined to be suitable reference genes for $\mathrm{BPH}$ resistance research. Ubiquitin was used as the reference gene for all of the qRT-PCR analysis in this study.

To find additional stable reference genes for the gene expression analysis under BPH-infestation conditions, 373 probe sets with total variance $<5 \%$ in the microarray data were screened. Two probe sets with low expressional variances and appropriate expressional levels were selected, Os.1322.1.S1_at (Os05g23860) and Os.145.1.S1_ a_at (Os02g56000) (Fig. 2), and the genes corresponding to these two probe sets were annotated to be rab GDP dissociation inhibitor alpha and $26 \mathrm{~S}$ protease regulatory subunit 6A, respectively, in the MSU Rice Genome Annotation Project Release 7 (http://rice.plantbiology.msu.edu/index.shtml). They were also stably expressed during the entire life cycles of the indica rice varieties 'Minghui 63' and 'Zhenshan 97' [23].

\section{Different defense responses, at the genome level, between RH and TN1 to BPH infestation}

At $6 \mathrm{~h}$ after needle puncturing, there were 92 differential probe sets in RH compared with the samples under natural growth, while there were 211 in TN1, indicating TN1's more sensitive reaction to simple mechanical damage than $\mathrm{RH}$. However, at $24 \mathrm{~h}$ after needle puncturing, fewer differential probe sets were detected in both RH (27) and TN1 (38) (Table 1), indicating that the

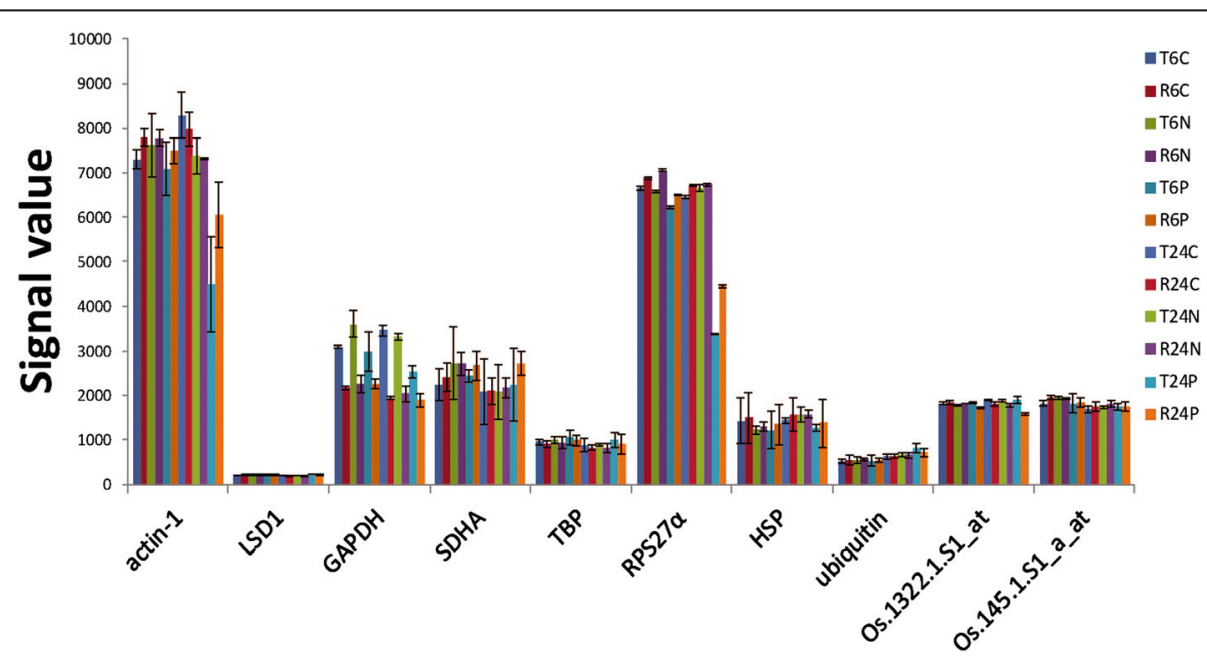

Fig. 2 Signal values of actin-1, LSD1, GAPDH, SDHA, TPB, RPS27a, HSP, Ubiquitin, Os.1322.1.S1_at and Os.145.1.S1_a_at in the microarray data. Among the eight frequently-used reference genes, TBP and Ubiquitin were more stably expressed than the others under BPH infestation. Os.1322.1.S1_at (Os05923860) and Os.145.1.S1_a_at (Os02g56000) are two newly identified references with stable expressions under BPH infestation. Error bars indicate standard deviations of three biological replicates 
Table 1 Number of differentially-expressed probe sets detected in $\mathrm{RH}$ and TN1 under needle-puncturing and $\mathrm{BPH}$-infestation treatments

\begin{tabular}{|c|c|c|c|c|c|}
\hline \multirow[t]{2}{*}{ Comparison } & \multicolumn{2}{|c|}{$F C^{a}>2$} & \multicolumn{2}{|c|}{$F C^{a}>5$} & \multirow[t]{2}{*}{ Total } \\
\hline & Up & Down & Up & Down & \\
\hline R6N/R6C & 69 & 10 & 13 & 0 & 92 \\
\hline T6N/T6C & 164 & 21 & 26 & 0 & 211 \\
\hline R6P/R6C & 126 & 48 & 33 & 2 & 209 \\
\hline T6P/T6C & 151 & 10 & 12 & 0 & 173 \\
\hline R24N/R24C & 26 & 0 & 1 & 0 & 27 \\
\hline T24N/T24C & 31 & 3 & 4 & 0 & 38 \\
\hline R24P/R24C & 405 & 136 & 72 & 1 & 614 \\
\hline T24P/T24C & 1368 & 1639 & 301 & 48 & 3356 \\
\hline
\end{tabular}

${ }^{\mathrm{a}} \mathrm{FC}$ indicates the fold change of differential probe sets under different treatments

reactions to simple mechanical damage had almost recovered by $24 \mathrm{~h}$.

At $6 \mathrm{~h}$ after $\mathrm{BPH}$ infestation, there were more differential probe sets in RH (209) than in TN1 (173). The number of differential probe sets with high FCs $(>5)$ was 35 in RH and 12 in TN1 (Table 1). Although TN1 was relatively more sensitive to simple mechanical damage, more differential probe sets were detected in $\mathrm{RH}$ at $6 \mathrm{~h}$ (early stage) after $\mathrm{BPH}$ infestation, implying that the defense responses to $\mathrm{BPH}$ infestation in $\mathrm{RH}$ was more intensive than in TN1. At $24 \mathrm{~h}$ after BPH infestation, more differential probe sets (614) were detected in $\mathrm{RH}$ compared with at $6 \mathrm{~h}$, and the number of probe sets with FCs $>5$ also increased from 35 to 73, indicating that the signals of $\mathrm{BPH}$ infestation were progressively transmitted to the related downstream genes. However, $24 \mathrm{~h}$ after $\mathrm{BPH}$ infestation, the number of differential probe sets in TN1 dramatically increased to 3356, and the number with FCs $>5$ also increased dramatically from 12 to 349 (Table 1). Thus, more differential probe sets were detected in $\mathrm{RH}$ at $6 \mathrm{~h}$ after $\mathrm{BPH}$ infestation; however, many more differential probe sets were detected in TN1 at $24 \mathrm{~h}$ after $\mathrm{BPH}$ infestation. Many of the differential probe sets detected in TN1 at $24 \mathrm{~h}$ after $\mathrm{BPH}$ infestation may have represented the responses to wounding and various physiological stresses resulting from the serious damage caused by the BPH, instead of a resistance response. In $\mathrm{RH}$, the majority of differential probe sets were up-regulated at either 6 or $24 \mathrm{~h}$ after BPH infestation, indicating that the inducible expression of resistance-related genes may be the main molecular basis of the defense responses in $\mathrm{RH}$ to $\mathrm{BPH}$.

The Venn diagram analysis of differential probe sets is shown as Fig. 3. Among the differential probe sets of RH at $6 \mathrm{~h}$ after BPH infestation, 53 were also present at $6 \mathrm{~h}$ after needle puncturing. Among the differential probe sets of TN1 at $6 \mathrm{~h}$ after BPH infestation, 60 were also present at $6 \mathrm{~h}$ after needle puncturing. At $24 \mathrm{~h}$ after the treatments, most of the differential probe sets under needle puncturing, in either $\mathrm{RH}$ or $\mathrm{TN} 1$, were also present under $\mathrm{BPH}$ infestation. By excluding the differential probe sets of $\mathrm{RH}$ and TN1 under needle puncturing and those of TN1 under $\mathrm{BPH}$ infestation, a total of $192 \mathrm{RH}$-specific and $\mathrm{BPH}$-inducible probe sets were determined. These 192 probe sets may represent the basis of the inducible defense in $\mathrm{RH}$ to $\mathrm{BPH}$ (Additional file 3).

\section{Gene Ontology (GO) analysis}

The differential probe sets of RH and TN1 under needle puncturing (the differential probe set groups of R6N/ $\mathrm{R} 6 \mathrm{C}$ and T6N/T6C) or BPH infestation (groups of R6P/ R6C, R24P/R24C, T6P/T6C and T24P/T24C) were subjected to GO analysis using GOEAST software, which includes two principal GO categories: biological process (BP) and molecular function (MF) (Additional file 4). The GO analysis of the groups R24N/R24C and T24N/ T24C was not conducted because very few differential probe sets were identified at $24 \mathrm{~h}$ after needle puncturing. The first level of BP categories and MF categories with significant differences and the percentages of related differential probe sets with annotations are shown in Fig. 4. As expected, in the needle-puncturing groups (R6N/R6C and T6N/T6C), 'response to wounding' was the GO term found having significant differences. Of the BP categories, the differential probe sets of 'response to biotic stimulus', 'response to abiotic stimulus', 'response to wounding,' 'defense response to fungus', 'cell wall macromolecule catabolic' and 'chitin catabolic process' were mainly up-regulated, while those of 'lipid transport' were mainly down-regulated (Fig. 4a). Of the MF categories, the differential probe sets of resistance-related projects, such as 'serine-type endopeptidase inhibitor activity', 'chitinase activity,',beta-glucosidase activity' and 'tetrapyrrole binding' were mainly up-regulated, while those of 'RNA methyltransferase activity' were mainly downregulated (Fig. 4b).

\section{Seeking BPH resistance-related gene candidates in RH}

Because too many differential probe sets were identified to test them individually in this study (Fig. 1), strategies were employed to reduce the number of BPH resistancerelated gene candidates. One strategy was to combine the microarray analysis with QTL mapping. Four $\mathrm{BPH}$-resistance QTLs were previously reported [25-27] (Additional file 5). In this study, we identified 106 differential genes between $\mathrm{RH}$ and TN1 that were located within the four BPH-resistance QTL regions (Additional file 6), and 14 of them are likely to be associated with the $\mathrm{BPH}$ resistance according to their functional annotations (Table 2). Among them, five genes were located in 

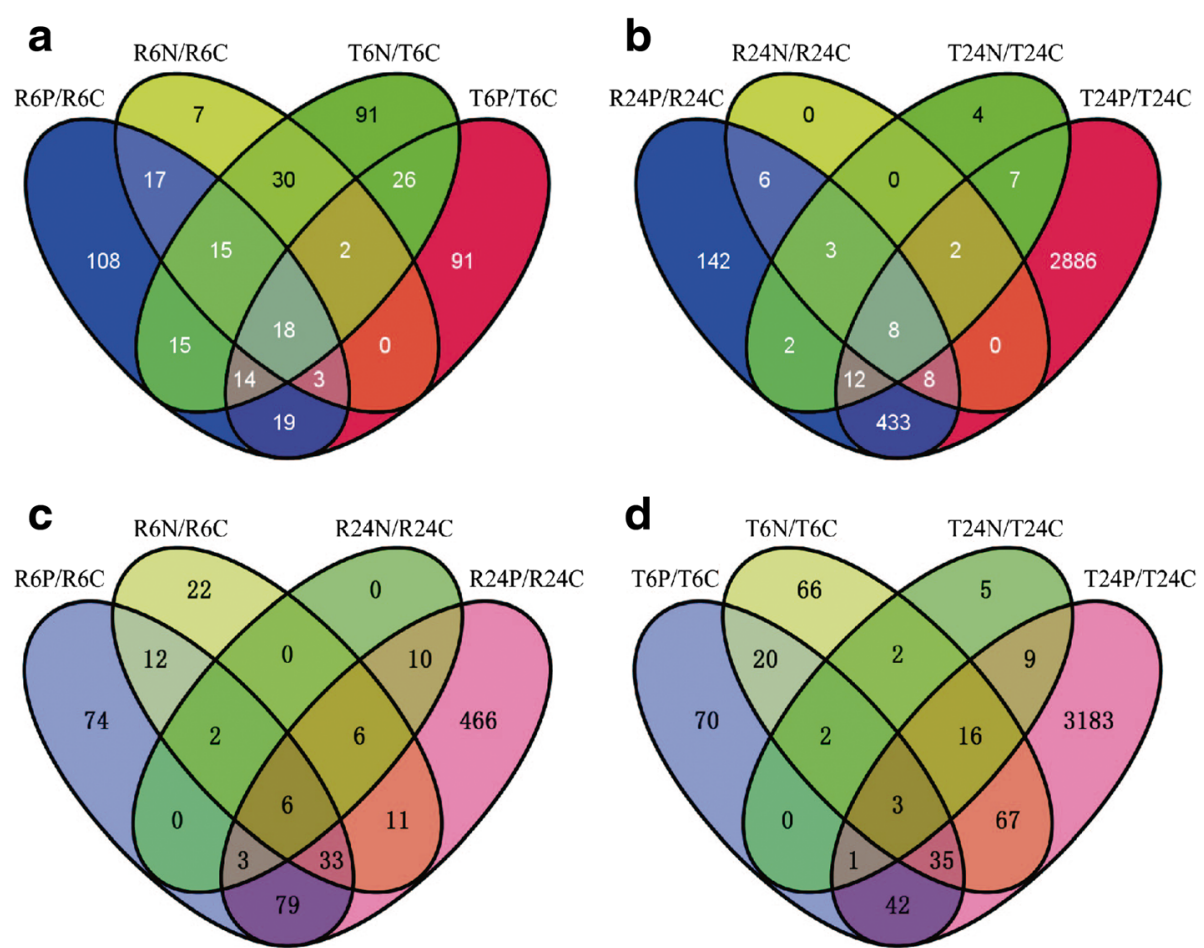

Fig. 3 Venn diagram analysis of differential probe sets under needle-puncturing and BPH infestation treatments at different times. a $6 \mathrm{~h}$ after needle puncturing or BPH infestation in RH and TN1; $\mathbf{b} 24 \mathrm{~h}$ after needle puncturing or BPH infestation in RH and TN1; 6 and $24 \mathrm{~h}$ after needle puncturing or $\mathrm{BPH}$ infestation in $\mathrm{RH}$; and $\mathbf{d} 6$ and $24 \mathrm{~h}$ after needle puncturing or $\mathrm{BPH}$ infestation in TN1

the Qbph3 region, five in Bph17, one in Bph3 and three in the Qbph10 region. A major BPH-resistance QTL, $B P H 17 / B P H 3$, which was mapped in the Bph17 region, was cloned and contains three tandem genes encoding lectin receptor kinases (OsLecRK1-OsLecRK3) [9]. However, the expression levels of these three genes were too low to be detected in this study.
The second strategy was to determine BPH resistancerelated gene candidates by examining gene expression patterns. Clustering the gene expression patterns divided the differential probe sets into 20 groups (Fig. 2). Group $\mathrm{m}$ represented those differential probe sets that were BPH-inducible only in RH but not in TN1. A Venn diagram analysis also helped to remove those differential
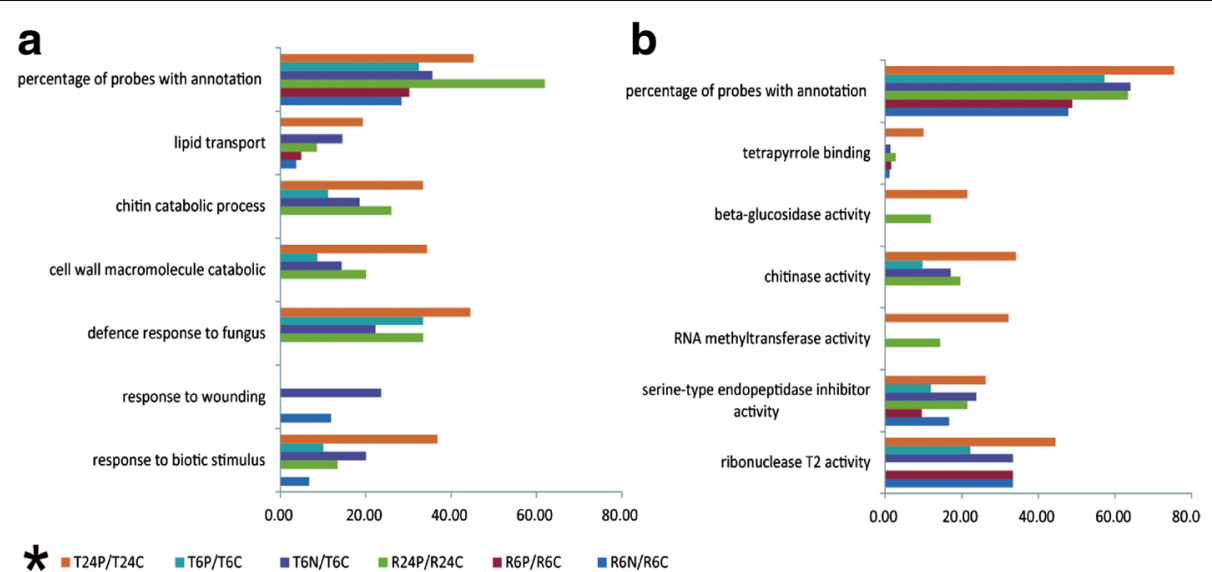

Fig. 4 Percentage of differential probe sets with annotations in the T24P/T24C, T6P/T6C, T6N/T6C, R24P/R24C, R6P/R6C and R6N/R6C groups. a biological process; and $\mathbf{b}$ molecular function 
Table 2 Fourteen selected candidate genes in the four $\mathrm{BPH}$-resistance QTLS of $\mathrm{RH}$

\begin{tabular}{lll}
\hline Candidate gene & QTL & Gene annotation \\
\hline Os03g12260 & Qbph3 & Cytochrome P450 family protein, expressed \\
Os03g12500 & Qbph3 & Cytochrome P450 74A2, putative, expressed \\
Os03g12660 & Qbph3 & Cytochrome P450 family protein, expressed \\
Os03g13370 & Qbph3 & TPR Domain containing protein, expressed \\
Os03g16740 & Qbph3 & $\begin{array}{l}\text { Protein kinase APK1B, chloroplast precursor, } \\
\text { expressed }\end{array}$ \\
Os04g08390 & Bph17 & Leucine Rich Repeat family protein, expressed \\
Os04g09920 & Bph17 & Cytochrome P450 family protein, expressed \\
Os04g11780 & Bph17 & resistance protein LR10, putative \\
Os04g14220 & Bph17 & NB-ARC domain containing protein, expressed \\
Os04g15650 & Bph17 & Leucine Rich Repeat family protein, expressed \\
Os06g04080 & Bph3 & Glycosyl hydrolases family 17 protein, expressed \\
Os10g39930 & Qbph10 & Cytochrome P450 family protein, expressed \\
Os10g41290 & Qbph10 & Protein kinase PVPK-1, putative, expressed \\
Os10g41550 & Qbph10 & Glycosyl hydrolase family 14 protein, expressed \\
\hline
\end{tabular}

probes sets induced by both mechanical damage and BPH infestation (Fig. 3). Finally, 23 gene candidates were selected based on the expression pattern clustering and Venn diagram analysis. These selected gene candidates shared a similar expression pattern (Fig. 5). They were inducible by $\mathrm{BPH}$ infestation only in $\mathrm{RH}$, but not by mechanical damage or in TN1. Among the $23 \mathrm{BPH}$ resistance-related gene candidates, Os03g12660, which encodes a cytochrome $\mathrm{P} 450$ protein, was the only gene also located in a BPH resistance QTL region (Qbph3).

\section{Dynamic changes of SA and JA levels and differential genes associated with SA, JA and ET pathways under BPH infestation}

The SA and JA levels (free status) in RH and TN1 were determined at 6,24 and $48 \mathrm{~h}$ after $\mathrm{BPH}$ infestation. Overall, the SA level in both $\mathrm{RH}$ and TN1 increased significantly after BPH infestation (Fig. 6a); while the JA level in both RH and TN1 decreased significantly after $\mathrm{BPH}$ infestation (Fig. 6b). Moreover, the change in the SA content in $\mathrm{RH}$ occurred more rapidly than that in TN1. The SA content in RH had increased at $6 \mathrm{~h}$ after $\mathrm{BPH}$ infestation, while that in TN1 did not increase until 24 h (Fig. 6a).

All of the differential genes under BPH-infestation treatment involved in the SA, JA and ET pathways are listed in Table 3. The information concerning the genes involved in the SA, JA and ET pathways refer to Kyoto Encyclopedia of Genes and Genomes annotations. Six differential genes involved in SA metabolism were detected, and they were up-regulated, except for one gene (Os02g19970) that was involved in both ET and SA metabolism.
Among 42 JA pathway-related genes, 17 were upregulated under $\mathrm{BPH}$ infestation in either $\mathrm{RH}$ or TN1. However, the expression level patterns of differential genes involved in the JA pathway were diverse between $\mathrm{RH}$ and TN1 (Table 3). All six differential genes in RH were up-regulated and were jasmonate ZIM-domain (JAZ) transcription factors, which are important negative regulators in the JA signal transduction pathway, implying that the JA pathway was repressed in RH under BPH infestation (Table 3). All 11 differentially expressed genes only presented in TN1 under BPH infestation were JA metabolism-related genes, including several JA synthesis-related lipoxygenase (LOX) genes. Additionally, 16 differential genes related to ET metabolism were detected. Most of them were present in TN1 and were down-regulated at $24 \mathrm{~h}$ after $\mathrm{BPH}$ infestation (Table 3). Comparatively, the ET pathway in $\mathrm{RH}$ was less affected after BPH infestation.

\section{Isolating BPH-inducible promoters}

Microarray analysis facilitates the isolation of $\mathrm{BPH}-$ inducible promoters. Six representative $\mathrm{BPH}$-inducible genes were selected from $23 \mathrm{BPH}$ resistance-related gene candidates (Fig. 5). The expression levels of these six genes were only induced by $\mathrm{BPH}$ infestation in $\mathrm{RH}$, and they were only slightly, or not at all, induced by needle puncturing or in TN1. A qRT-PCR analysis showed that these six genes were also up-regulated by a SA or JA treatment (Fig. 7).

The genomic sequences, including the promoter regions and coding sequences of these six selected $\mathrm{BPH}-$ inducible genes, were isolated by PCR from both $\mathrm{RH}$ and TN1, and then subjected to sequencing. No significant sequence differences in the promoter regions of these six genes were found between RH and TN1. Furthermore, a DNA sequence comparison of the coding regions showed no differences between $\mathrm{RH}$ and TN1. It was hypothesized that the inducibility of these genes in $\mathrm{RH}$ was not determined by the promoter sequences, but by the genetic background.

Additional rice varieties, including four $\mathrm{BPH}$-resistant ('PTB33', 'R644', 'B5' and 'IR54751') and four BPHsusceptible varieties ('Bai56,' 'Nipponbare,' 'Zhenshan97B' and ' 9311 '), were used for further examination. Similar to in $\mathrm{RH}$ and $\mathrm{TN} 1$, the expression levels of the six selected genes were relatively high and significantly induced by BPH infestation in all of the resistant varieties, but was relatively low and barely induced by BPH infestation in all of the susceptible varieties (Additional file 7). The genomic sequences of these six resistance-related genes were also isolated from all eight additional rice varieties and then compared. Neither the promoters nor the coding sequences had any significant sequence differences between the resistant and susceptible varieties. 

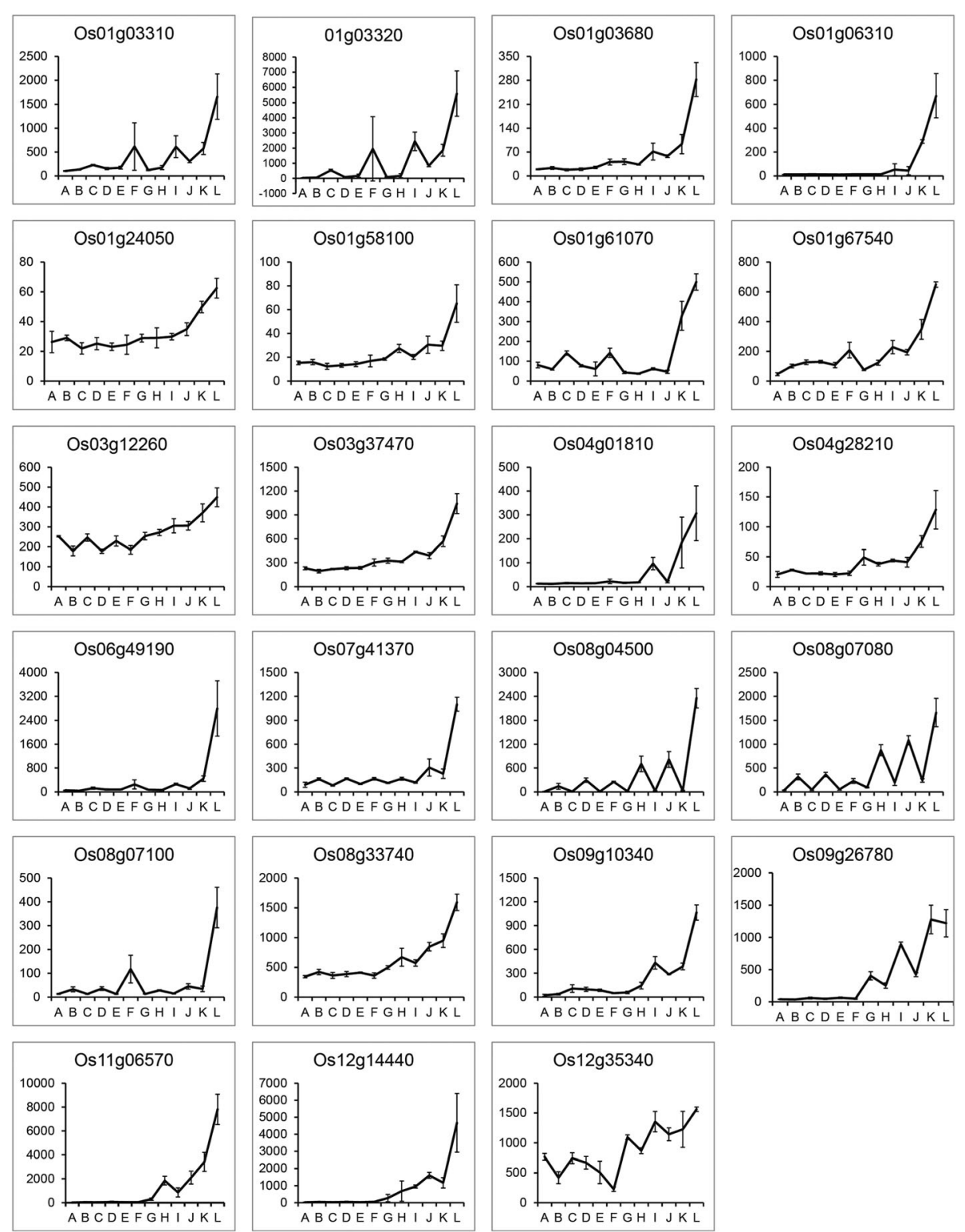

Fig. 5 Expression patterns of 23 selected BPH resistance-related gene candidates. These gene candidates share a similar expression pattern, which was inducible by BPH infestation only in RH but not by mechanical damage or in TN1. The six genes in boldface were selected for further detection. A-L on the $x$-axis represent T6C, R6C, T24C, R24C, T6N, R6N, T24N, R24N, T6P, R6P, T24P and R24P, respectively. Error bars indicate standard deviations of three biological replicates

\section{Discussion}

In this study, the whole genome-wide responses of the resistant $\mathrm{RH}$ and the susceptible TN1 under BPH infestation were determined and compared through a microarray analysis. A similar study reported by Wang et al., conducted a microarray analysis using $\mathrm{RH}$ and TN1 as the rice materials under infestation [28, 29]. However, the study of Wang et al. did not incorporate an untreated control for each sampling time point $(0,8$ and $24 \mathrm{~h}$ after $\mathrm{BPH}$ infestation), and they simply used the samples collected at $0 \mathrm{~h}$ as the control for the microarray analysis. This design is problematic because the expression levels of many genes are regulated by a circadian rhythm or other environmental factors. The expression pattern clustering in our study clearly showed that 3793 differential probe sets, such as groups l, q, r and $\mathrm{s}$ in Fig. 2, accounting for almost a half of all detected differential probe sets, were obviously affected by a circadian rhythm or other environmental factors. These were possibly mistaken for having an association with BPH infestation because of the lack of a control for each sampling time. Second, our study included the 

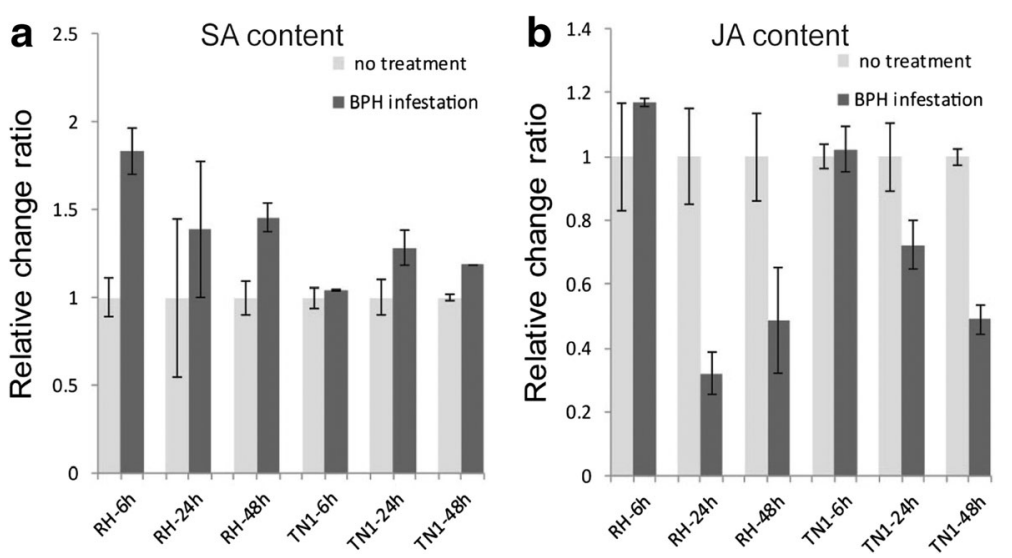

Fig. 6 SA and JA relative change ratios in RH and TN1 under BPH infestation. a SA relative change ratios in RH and TN1; b JA relative change ratios in $\mathrm{RH}$ and TN1. Error bars indicate standard deviations of three biological replicates

needle-puncturing treatment, while the study of Wang et al. did not. The immune responses of rice to $\mathrm{BPH}$ involve molecular interactions between rice and $\mathrm{BPH}$, and the resistance reactions of rice are triggered by specific effectors in BPH saliva, which are delivered into rice cells by feeding and are recognized by specific receptors in rice [30]. The needle-puncturing treatment can be used to exclude those differential genes that respond to simple mechanical wounding.

There are several other studies that also profiled gene expression in rice varieties responding to $\mathrm{BPH}$ infestation using RNA-sequencing (RNA seq) or microarray analyses [31, 32]. In the study of Lv et al., RNA-seq of BPH susceptible WT '9311' and resistant '9311' introgression lines containing $B P H 15$ was conducted to help identify candidate genes of $B P H 15$, which are located in a recombination cold spot [31]. The study of Wang et al. compared gene expression profiles between $B t$ and non$B t$ rice in response to $\mathrm{BPH}$ infestation [32]. In these two studies, the genetic backgrounds of rice materials used for the gene expressional analyses were both BPH susceptible '9311' [31] or 'Xiushui 11' [32]; therefore, their results could not present the responses of a rice variety resistant to $\mathrm{BPH}$ infestation .

The microarray analysis showed significant response differences between $\mathrm{RH}$ and TN1 to BPH infestation. The response of $\mathrm{RH}$ was more active at the early stage of $\mathrm{BPH}$ infestation compared with TN1 because more differential probe sets (209) were detected in $\mathrm{RH}$ at $6 \mathrm{~h}$ after BPH infestation than in TN1 (173). In contrast, many more differential probe sets (3356) were detected in TN1 at $24 \mathrm{~h}$ after BPH infestation than in RH (614). Many of the differential probe sets presented at $24 \mathrm{~h}$ reflected the physiological and metabolic changes in TN1 under BPH attack caused by its lack of resistance. The GO analysis also confirmed that many of the differential probe sets in $\mathrm{TN} 1$ at $24 \mathrm{~h}$ after $\mathrm{BPH}$ infestation were related to abiotic and biotic stresses. Most differential probe sets detected in $\mathrm{RH}$ at either 6 or $24 \mathrm{~h}$ after $\mathrm{BPH}$ infestation were up-regulated, implying that the up-regulation of the defense-related genes was possibly the molecular basis of $\mathrm{BPH}$ resistance in $\mathrm{RH}$.

The change in the SA level in RH occurred more rapidly than in TN1, and the increase in the SA level occurred prior to the decrease in the JA level, indicating that the SA pathway plays a leading role in triggering the BPH-resistance response. The decrease in the JA content was probably caused by the antagonist effect of $\mathrm{SA}$, which increased after $\mathrm{BPH}$ infestation. The upregulation of SA synthesis-related genes was the main change trend in the SA pathway, which was consistent with the change in the SA content after BPH infestation. However, differential genes in the JA pathway showed significant diverse change patterns between $\mathrm{RH}$ and TN1. In RH, all six differential genes were up-regulated and were JAZ transcription factors, which are important negative regulators of the JA pathway. While in TN1, 11 differential genes involved in JA synthesis were upregulated and only two were JAZ transcription factors. JA is also associated with wounding responses. As a susceptible variety, TN1 lacks BPH resistance-related genes and undergoes much more serious damage under $\mathrm{BPH}$ infestation. Therefore, the up-regulation of JA synthesis-related genes probably represented a response to wounding or another stress caused by $\mathrm{BPH}$, instead of a resistance response.

Although the four major BPH resistance QTLs were cloned by map-based techniques, many BPH resistancerelated genes, including OsHI-LOX [12], Bphi008a [13], OsERF3 [14], OsPLD $\alpha 4$ and $\alpha 5$ [15], OsACS2 [17], Osr9LOX1 [19], and OsIMT1 [20], were also isolated using a reverse genetics strategy. Almost all of these $\mathrm{BPH}$ resistance-related genes are inducible to $\mathrm{BPH}$ or SSB infestation, and are involved in SA, JA, or ET pathways. A 
Table 3 Differentially-expressed genes involved in the SA, JA and ET pathways during BPH infestation

\begin{tabular}{|c|c|c|c|c|c|}
\hline \multirow[t]{2}{*}{ Gene ID } & \multicolumn{4}{|c|}{ Fold changed (under BPH infestation) } & \multirow[t]{2}{*}{ Gene annotation } \\
\hline & R6P/R6C & R24P/R24C & T6P/T6C & $\mathrm{T} 24 \mathrm{P} / \mathrm{T} 24 \mathrm{C}$ & \\
\hline \multicolumn{6}{|l|}{ SA pathway(59) } \\
\hline Os02g19970 & 0.41 & - & - & - & aminotransferase, classes I and II, domain containing protein, OsNAAT2 \\
\hline Os03g13210 & - & - & 9.43 & - & peroxidase precursor \\
\hline Os04g43800 & 3.08 & 2.44 & - & - & phenylalanine ammonia-lyase, OsPAL6 \\
\hline Os08g02110 & 3.57 & 3.29 & 5.12 & - & peroxidase precursor \\
\hline Os08g34790 & - & - & 4.08 & - & AMP-binding domain containing protein, Os $4 C L 5$ \\
\hline Os11g02130 & 2.07 & - & 2.60 & - & peroxidase precursor \\
\hline \multicolumn{6}{|l|}{ JA pathway(42) } \\
\hline Os01g06600 & - & - & 2.50 & - & glutaryl-CoA dehydrogenase, mitochondrial precursor \\
\hline Os01g55650 & - & - & 2.00 & - & phospholipase, patatin family, OspPLAIVa \\
\hline Os02g10120 & - & - & 3.25 & - & lipoxygenase, OsLOX1 \\
\hline Os02g17390 & - & - & 2.07 & - & 3-hydroxyacyl-CoA dehydrogenase \\
\hline Os05g07090 & - & - & 4.93 & - & acyl-coenzyme A dehydrogenase, mitochondrial precursor \\
\hline Os06g11210 & - & - & 4.09 & - & 12-oxophytodienoate reductase \\
\hline Os08g39840 & - & - & 6.92 & - & lipoxygenase, chloroplast precursor, OsLOX9 \\
\hline Os08g39850 & - & - & 6.19 & - & lipoxygenase, chloroplast precursor, OsLOX8 \\
\hline Os11g39220 & - & - & 2.37 & - & acyl-coenzyme A oxidase, OsACX2 \\
\hline Os12g26290 & - & - & 2.93 & - & alpha-DOX2 \\
\hline Os12g37260 & - & - & 3.64 & - & lipoxygenase 2.1, chloroplast precursor, OsLOX11 \\
\hline Os03g08310 & 3.32 & 5.89 & - & - & ZIM domain containing protein, OsJAZ9; OsTIFY11a \\
\hline Os03g08320 & 8.16 & 8.55 & 13.67 & 4.82 & ZIM domain containing protein, OsJAZ11; OsTIFY11C \\
\hline Os03g08330 & 2.65 & 3.03 & - & - & ZIM domain containing protein, OsJAZ10; OsTIFY11b \\
\hline Os09g26780 & 4.82 & 3.15 & - & - & zinc-finger protein, OsJAZ8; OsTIFY10C \\
\hline Os10g25230 & 3.40 & 4.52 & - & - & ZIM domain containing protein, OsJAZ13; OsTIFY11e \\
\hline Os10g25290 & 3.21 & 2.73 & 2.93 & 3.12 & ZIM domain containing protein, OsJAZ12; OsTIFY11d \\
\hline \multicolumn{6}{|l|}{ ET pathway(82) } \\
\hline Os01g52260 & - & - & 2.85 & 2.10 & serine acetyltransferase protein \\
\hline Os01g59920 & - & - & 0.33 & - & cysteine synthase, chloroplast precursor \\
\hline Os01g74650 & - & - & 0.46 & - & cysteine synthase, mitochondrial precursor \\
\hline Os02g14110 & - & - & 0.47 & - & aminotransferase, classes I and II, domain containing protein \\
\hline Os02g19970 & - & - & 0.41 & - & aminotransferase, classes I and II, domain containing protein, OsNAAT2 \\
\hline Os02g53180 & - & - & 3.30 & - & 1-aminocyclopropane-1-carboxylate oxidase protein, OsACO3 \\
\hline Os03g25940 & 0.38 & - & - & - & cystathionine gamma-synthase \\
\hline Os03g55280 & - & - & 0.25 & - & semialdehyde dehydrogenase, NAD binding domain containing protein \\
\hline Os06g36880 & - & - & 2.22 & - & cysteine synthase \\
\hline Os07g08500 & - & - & 0.41 & - & C-5 cytosine-specific DNA methylase, OsMET1b \\
\hline Os07g22600 & - & - & 0.49 & - & spermidine synthase \\
\hline Os08g25390 & 0.47 & - & 0.22 & - & bifunctional aspartokinase/homoserine dehydrogenase, chloroplast precursor \\
\hline Os09g12290 & - & - & 0.41 & - & bifunctional aspartokinase/homoserine dehydrogenase, chloroplast precursor \\
\hline Os09g27750 & - & - & 2.27 & - & 1-aminocyclopropane-1-carboxylate oxidase 1, OsACO2; OsACO1 \\
\hline Os10g01570 & - & - & 0.24 & - & C-5 cytosine-specific DNA methylase, OsCMT3a \\
\hline Os10g37340 & 2.19 & 2.10 & 2.27 & - & cystathionine gamma-synthase \\
\hline
\end{tabular}



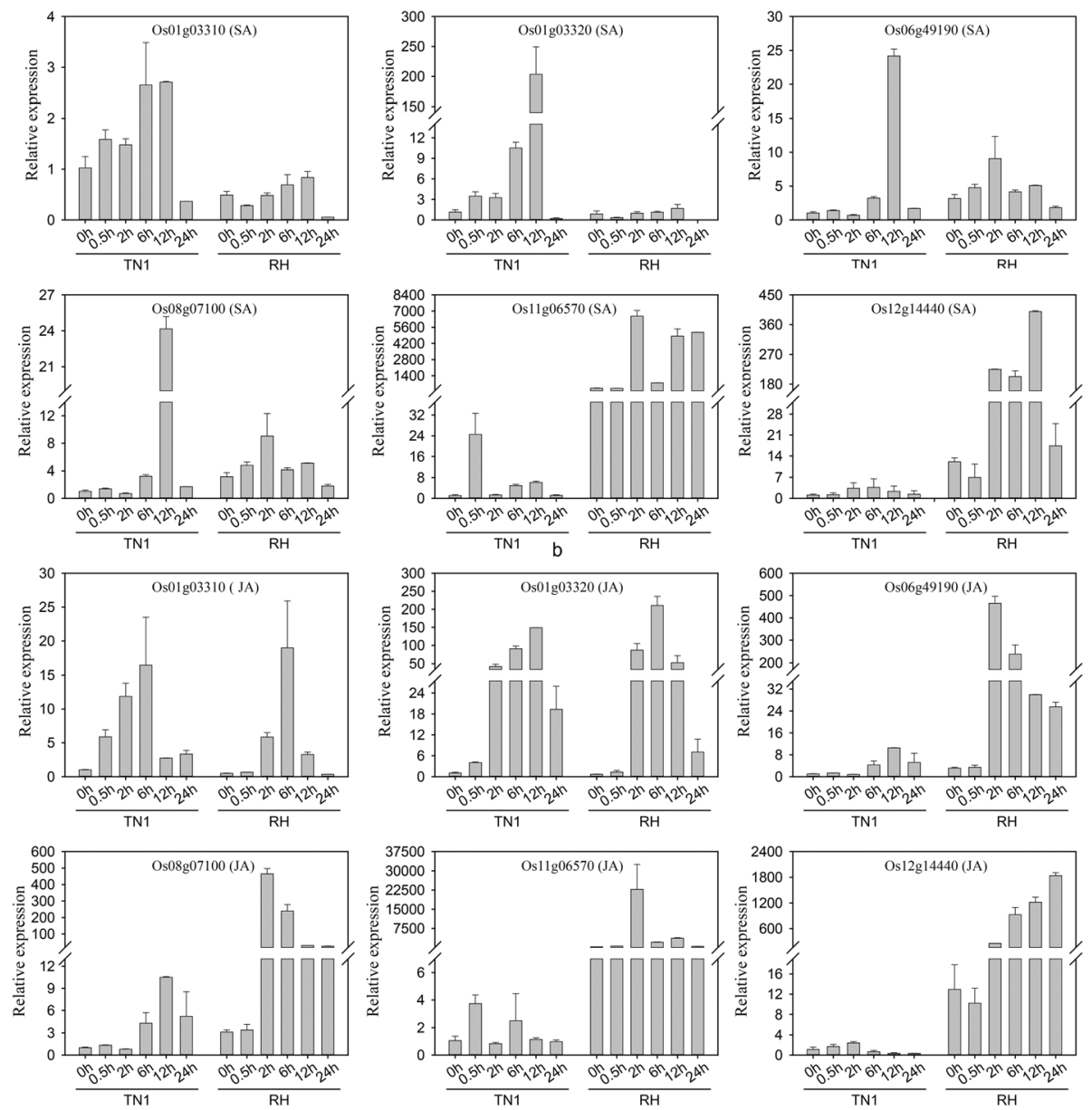

Fig. 7 Expression pattern testing of six selected BPH resistance-related genes in RH and TN1 after independent SA and JA treatments. All six genes were up-regulated after independent SA and JA treatments. The gene expression levels in TN1 without a treatment were normalized as the calibrators. Error bars indicate standard deviations of three biological replicates

cDNA microarray analysis of $\mathrm{RH}$ and TN1 under $\mathrm{BPH}$ infestation laid the foundations for identifying new $\mathrm{BPH}$ resistance-related genes.

$\mathrm{BPH}$-responsive promoters have potential applications in developing BPH-resistant genetically modified rice varieties. Therefore, the approximate $2-\mathrm{kb}$ upstream promoter regions of some representative $\mathrm{RH}$-specific and $\mathrm{BPH}$-inducible genes were isolated and fused with the GUS reporter gene. However, when tested in $\mathrm{BPH}-$ susceptible rice varieties, these putative $\mathrm{BPH}$-inducible promoters were either not, or were poorly, induced under $\mathrm{BPH}$ infestation. A DNA sequencing analysis showed that these $\mathrm{RH}$-specific and $\mathrm{BPH}$-inducible genes showed no significant sequence differences in both the promoter and coding regions between BPH-resistant and -susceptible rice varieties. Thus, the promoters of most of these $\mathrm{BPH}$-inducible genes were probably not $\mathrm{BPH}$-inducible, and they may be regulated by their upstream trans-regulators in the BPH-resistance network. In other words, their expression pattern was determined by the genetic background instead of the promoter sequence.

\section{Conclusions}

In this study, a cDNA microarray analysis was conducted to reveal the genome-wide response differences between RH and TN1 under BPH infestation. Expression pattern clustering of differential probe sets demonstrated that 1467 differential probe sets (Groups p and k) may be associated with the constitutive resistance of $\mathrm{RH}$ and 67 (Groups a and $\mathrm{m}$ ) with the $\mathrm{BPH}$ infestationresponsive resistance. The Venn diagram analysis determined $192 \mathrm{RH}$-specific and $\mathrm{BPH}$-inducible probe sets. Finally, 23 genes were selected as $\mathrm{BPH}$ resistance-related gene candidates based on the expression pattern clustering and Venn diagram analysis. The overall response of $\mathrm{RH}$ to $\mathrm{BPH}$ infestation was more prompt than that of TN1, at the global gene expression and the SA levels. In $\mathrm{RH}$, the significant increase in the SA (6 h after BPH infestation) occurred prior to the significant decrease in 
the JA (24 $\mathrm{h}$ after $\mathrm{BPH}$ infestation), implying a leading role of SA in mediating $\mathrm{BPH}$-resistance responses. In $\mathrm{RH}$, differential genes in the SA pathway were synthesisrelated and up-regulated after $\mathrm{BPH}$ infestation. The differential genes in the JA pathway were up-regulated and were JAZ transcription factors, which are important negative regulators of the JA pathway. These results were consistent with the changes in the SA and JA levels in $\mathrm{RH}$ seedlings infested with $\mathrm{BPH}$. Comparatively, genes involved in the ET pathway were less affected by a BPH infestation in $\mathrm{RH}$. The results of this study aid in understanding the molecular foundation of BPH resistance in the $\mathrm{RH}$ and facilitate the identification of new resistancerelated genes for breeding $\mathrm{BPH}$-resistant rice varieties.

\section{Methods}

\section{Plant materials}

Rice varieties RH and TN1 were kindly provided by Prof. Hongxia Hua. $\mathrm{RH}$ is an indica rice cultivar from Sri Lanka, with a high-level, broad-spectrum resistance to all $\mathrm{BPH}$ biotypes. The indica rice cultivar TN1 is highly susceptible to all BPH biotypes. Four BPH-resistant rice varieties 'PTB33,' 'R644,' 'B5' and 'IR54751' were identified and provided by Prof. Yuqing He. Four BPH-susceptible rice varieties 'Bai56,' 'Nipponbare', 'Zhenshan97B' and '9311' were previously collected and maintained in our laboratory. The $\mathrm{BPH}$ resistance of all involved rice materials were validated in our laboratory prior to the current research.

\section{Plant sample preparation for the microarray analysis}

Two rice varieties, $\mathrm{BPH}$-resistant $\mathrm{RH}$ and $\mathrm{BPH}$ susceptible TN1, were used. The BPH population for this experiment was collected from a local rice field in $\mathrm{Wu}-$ han, China, and raised on TN1 in cages. This BPH population is a mix of BPH biotypes 1 and 2, which represent the main natural $\mathrm{BPH}$ populations present locally.

Seeds of RH and TN1 were sown in small pots and grown under natural conditions. Approximately 15 seeds were sown per pot, and after 2 weeks only 10 wellgrown rice seedlings were retained in each pot. Three treatments, untreated (naturally grown), needle puncturing and BPH infestation, were implemented on the 2week-old RH and TN1 seedlings. For needle puncturing, each plant was pricked with a needle 15 times at the bottom of the seedling, and then grown normally. For BPH infestation, approximately 100 third-instar BPH nymphs were introduced per pot (an average of 10 nymphs per seedling). Each pot was placed in a single netted cage to prevent nymphs from escaping. Sampling time points of 6 and $24 \mathrm{~h}$ after BPH infestation were used for all of the treatments. There were three replications for each treatment and sampling time point. All 10 seedlings from each pot were collected as a replication. The seedling shoots (the aerial part) were collected after the treatment, immediately placed in liquid nitrogen and then stored at $-80{ }^{\circ} \mathrm{C}$. Finally, all of the samples were shipped to CapitalBio Corporation (Beijing, China) for the microarray determinations.

\section{RNA extraction, microarray hybridization and data analysis}

RNA extraction, purification, microarray hybridization and gene annotation were conducted by CapitalBio. After RNA extraction, the total RNA was purified using a Nucleospin RNA Clean-up kit (Macherey-Nagel, Germany) following the manufacturer's instructions. The RNA quality was determined by electrophoresis on $1 \%$ agarose gel with formaldehyde. Biotin-labeled cRNA was synthesized using a MessageAmp ${ }^{\text {st }}$ II-Biotin kit (Ambion, TX, USA). Under the guidance of the Affymetrix GeneChip Expression Analysis technical manual, the biotinlabeled cRNA was linearized into fragments of 35-300 bp in length. The linear cRNA was hybridized with an Affymetrix Rice Genome array in an Affymetrix GeneChip Hybridization Oven 320 at $45{ }^{\circ} \mathrm{C}$ for $16 \mathrm{~h}$. After hybridization, the chip was washed and dyed in an Affymetrix Fluidics Station 400. Finally, the chip was scanned using a GeneChip Scanner 3000. The Single Array Analysis, Comparison Analysis and Molecule Annotation System were performed by CapitalBio.

\section{Data analysis}

Venn diagrams were made on the website http://bioinfogp.cnb.csic.es/tools/venny/. Probe-set expression pattern clustering was performed using the Multi Experiment Viewer software in the java environment (http://mev.t $\mathrm{m} 4$.org/). The GO analysis was performed on the website http://omicslab.genetics.ac.cn/GOEAST/php/affymetrix.php?\#step1_anchor [33]. All of the expressiondifferential probes were previously identified by CapitalBio using a one-way ANOVA. The gene information used in our analysis was from the websites Kyoto Encyclopedia of Genes and Genomes (http://www.kegg.jp/kegg/kegg2.h tml), CREP (http://crep.ncpgr.cn/), GRASSIUS (http://gra ssius.org/grasstfdb.html) and RGAP (http://rice.plantbiolo gy.msu.edu/index.shtml).

\section{Expression profiling following plant hormone treatments}

The RH and TN1 plants were grown under greenhouse conditions at $25-30{ }^{\circ} \mathrm{C}$ and a photoperiod of $14 \mathrm{~h}$ light/ $10 \mathrm{~h}$ dark. At the trefoil stage, the RH and TN1 plants were transferred into hydroponic nutrient solutions containing either $0.1 \mathrm{mM} \mathrm{SA}$ or JA. At each time point of 0 , $0.5,2,6,12$ and $24 \mathrm{~h}$ after treatment, three to five whole plants were collected for RNA isolation and extraction. 


\section{qRT-PCR analysis}

RT with $2 \mu \mathrm{g}$ of DNase-treated total RNA was performed using a Transcriptor First Strand cDNA Synthesis Kit (Roche, Mannheim, Germany) following the manufacturer's instructions. The real-time PCR was conducted using SYBR Premix Ex Taq TM (Takara, Dalian, China). The reactions were prepared in a volume of $20 \mu \mathrm{L}$ containing $10 \mu \mathrm{L}$ of SYBR Premix Ex Taq TM (2×), $0.4 \mu \mathrm{M}$ for each gene-specific primer $(10 \mu \mathrm{M})$, $0.4 \mu \mathrm{L}$ of ROX Reference Dye II $(10 \mu \mathrm{M}), 2 \mu \mathrm{L}$ of cDNA template and $6.8 \mu \mathrm{L}$ of $\mathrm{ddH}_{2} \mathrm{O}$. The Ubiquitin gene was used as the reference gene. The real-time PCR was executed on a 7500 Real-time PCR System (Applied Biosystems, Foster City, CA, USA). Each RNA sample was used twice in technical replications. All of the primers used in this study are listed in Additional file 8.

\section{Hormone determinations}

SA and JA levels in plant tissues were measured by liquid chromatography-tandem mass spectrometry (LCMS/MS 8030 plus, Shimadzu, Beijing, China) as follows: a total of $200 \mathrm{mg}$ of fresh sample frozen in liquid nitrogen was well ground using a small glass pestle in a $2-\mathrm{mL}$ vial. Following the addition of $1.0 \mathrm{~mL}$ of $80 \%$ methanol, homogenates were well mixed in an ultrasonic bath and then kept overnight at $4{ }^{\circ} \mathrm{C}$. After being centrifuged at $15,200 \times g$ for $10 \mathrm{~min}$, the supernatant was collected and then vacuumed to dryness in a Jouan RCT-60 concentrator. Dried extract was dissolved in $200 \mu \mathrm{L}$ of $0.1 \mathrm{~mol} / \mathrm{L}$ sodium phosphate solution $(\mathrm{pH}$ 7.8) and later passed through a Sep-Pak C18 cartridge (Waters, MA, USA). The cartridge was eluted with $1.5 \mathrm{~mL}$ of $80 \%$ methanol, and the eluate was vacuumed to dryness again. After being dissolved in $10 \mathrm{~mL}$ of $10 \%$ methanol, $5 \mu \mathrm{L}$ of such solution was injected into the LC-MS/MS system. LC was performed using a $2.0 \mathrm{~mm}$ I.D. $\times 75 \mathrm{~mm}$ Shim-pack XR ODSI column $(2.2 \mu \mathrm{m}$, Shimadzu $)$ at a column temperature of $40{ }^{\circ} \mathrm{C}$. The mobile phase, containing solvent A $(0.02 \% \mathrm{v} / \mathrm{v}$ aqueous acetic acid) and solvent $\mathrm{B}$ (100\% v/v methanol), was employed in a gradient mode [time/A concentration/B concentration ( $\mathrm{min} / \% / \%)$ for $0 /$ 90/10; 5/10/90;6/10/90 and 6.1/90/10] at an eluent flow rate of $0.3 \mathrm{~mL} / \mathrm{min}$. For $\mathrm{SA}$, the mass system was set to multiple reactions monitoring mode using electrospray ionization in the negative ion mode. The operation conditions used were a nebulizing gas flow of $2.5 \mathrm{~L} / \mathrm{min}$, drying gas flow of $15 \mathrm{~L} / \mathrm{min}$, desolvation temperature of $150{ }^{\circ} \mathrm{C}$ and heat block temperature of $400{ }^{\circ} \mathrm{C}$. The ionization conditions were pre-bias voltages of $10 \mathrm{~V}$ for quadrupole 1 and $24 \mathrm{~V}$ for quadrupole 3, collision energy of $28 \mathrm{eV}$, and mass-to-charge ratio of 137/93. For JA, the mass system was set to multiple reactions monitoring mode using electrospray ionization in the negative ion mode. The operation conditions used were a nebulizing gas flow of $3 \mathrm{~L} / \mathrm{min}$, drying gas flow of $15 \mathrm{~L} / \mathrm{min}$, desolvation temperature of $250{ }^{\circ} \mathrm{C}$ and heat block temperature of $500{ }^{\circ}$ C. The ionization conditions were pre-bias voltages of $10 \mathrm{~V}$ for quadrupole 1 and $10 \mathrm{~V}$ for quadrupole 3, collision energy of $15 \mathrm{eV}$, and mass-to-charge ratio of 209/59.

\section{Additional files}

\begin{abstract}
Additional file 1: Raw data of all detected probe sets $(57,194)$ in 36 cDNA chips (https://www.ncbi.nlm.nih.gov/geo/, GEO accession number: GSE74106). (TXT 1023 kb)
\end{abstract}

Additional file 2: Information on the 8019 differentially-expressed probe sets detected in this study. (XLSX $258 \mathrm{~kb}$ )

Additional file 3: Information on $192 \mathrm{RH}$-specific and $\mathrm{BPH}$-inducible differentially-expressed probe sets. (XLSX $12 \mathrm{~kb}$ )

Additional file 4: $\mathrm{GO}$ analysis of differential probe sets between $\mathrm{RH}$ and TN1 under needling puncturing and BPH infestation conditions. (XLSX 183 kb)

Additional file 5: Four rice BPH-resistance QTLs in chromosomes 3, 4, 6 and 10 of $\mathrm{RH}$ that were previously reported. (JPG $161 \mathrm{~kb}$ )

Additional file 6: The 103 potential resistance-related genes in the four $\mathrm{BPH}$-resistance QTL regions in $\mathrm{RH}$. (XLSX $15 \mathrm{~kb}$ )

Additional file 7: Expression pattern analysis of six selected $\mathrm{BPH}$-induced genes in 10 rice varieties. The gene expression levels in TN1 with no treatment were normalized as the calibrators. Error bars indicate the standard deviations of three biological replicates. The expression levels of all the six selected genes were relatively high and significantly induced by $\mathrm{BPH}$ infestation $(24 \mathrm{~h})$ in the resistant varieties $\mathrm{RH}$, 'PTB33', 'R644', 'B5' and 'IR54751', but were relatively low and barely induced by BPH infestation in the susceptible varieties TN1, 'Bai56', 'Nipponbare', 'Zhenshan 97B' and 9311. (JPG 2037 kb)

Additional file 8: All of the primers used in this study. (DOCX $22 \mathrm{~kb}$ )

\section{Abbreviations}

BPH: Brown planthopper; ET: Ethylene; GO: Gene Ontology; JA: Jasmonic acid; JAZ: Jasmonate ZIM-domain protein; QTL: Quantitative trait locus; RH: Rathu Heenati; SA: Salicylic acid; TN1: Taichung Native 1

\section{Acknowledgements}

The authors would like to thank National Special Key Project for Transgenic Breeding (2016ZX08010002-002), Project 2013PY06 supported by

Fundamental Research Funds for the Central University, and National Science Foundation of China (91317312)

\section{Funding}

National Special Key Project for Transgenic Breeding (2016ZX08010002-002) to HC funded microarray detection, data analysis, qRT-PCR, and DNA sequencing. Project 2013PY06 supported by Fundamental Research Funds for the Central University to $\mathrm{HC}$ supported collection and identification of rice materials. National Science Foundation of China (91317312) to LX supported plant hormone determination.

\section{Availability of data and materials}

Data from this study are available from the NCBI's Gene Expression Omnibus through GEO Series accession number GSE81297 (https:/www.ncbi.nlm.nih.gov/geo/).

\section{Authors' contributions}

$\mathrm{YL}$ and $\mathrm{HC}$ conceived and designed the research; $\mathrm{CL}, \mathrm{CL}$ and $\mathrm{ZZ}$ prepared the samples; $C L$ and $L X$ finished hormone determinations; $C L, F L$ and RW performed the analysis; $\mathrm{CL}$ and $\mathrm{HC}$ wrote the manuscript. All authors have read and approved the final version of this manuscript.

\section{Competing interests}

The authors declare that they have no competing interests.

Consent for publication

Not applicable. 


\section{Ethics approval and consent to participate}

Not applicable.

\author{
Author details \\ ${ }^{1}$ National Key Laboratory of Crop Genetic Improvement and National Centre \\ of Plant Gene Research (Wuhan), Huazhong Agricultural University, Wuhan \\ 430070, China. ${ }^{2}$ Hunan Provincial Key Laboratory of Phytohormones and \\ Growth Development, Hunan Agricultural University, Changsha 410128, \\ China.
}

Received: 6 October 2016 Accepted: 23 February 2017

Published online: 28 February 2017

\section{References}

1. Normile D. Agricultural research. Reinventing rice to feed the world. Science. 2008;321(5887):330-3.

2. Cha YS, Ji H, Yun DW, Ahn BO, Lee MC, Suh SC, et al. Fine mapping of the rice $B p h 1$ gene, which confers resistance to the brown planthopper (Nilaparvata lugens Stål), and development of STS markers for markerassisted selection. Mol Cells. 2008;26(2):146-51.

3. Reifman VG, Pinsker NI, Rivera $\subset$. Grassy stunt disease of rice and its transmission by the planthopper Nilaparvata Lugens Stal [J]. Plant Dis Report. 1966;50:453-56.

4. Ling KC, Tiongco ER, Aguiero VM. Transmission of rice ragged stunt disease. Int Rice Res Newsl. 1977;2(6):11-2.

5. Wu H, Liu Y, He J, Liu Y, Jiang L, Liu L, et al. Fine mapping of brown planthopper (Nilaparvata lugens Stål) resistance gene Bph28(t) in rice (Oryza sativa L.). Mol Breed. 2014;33(4):909-18.

6. Sharma PN, Mori N, Takumi S, Nakamura C. Conventional and molecular studies of brown planthopper (Nilaparvata lugens Stål) resistance genes in rice: a basis for future study of natural insect resistance genes using molecular markers in nepal. Nepal J Sci Technol. 2015;15(1):145-56.

7. Hu J, Xiao C, Cheng M, Gao G, Zhang Q, He Y. Fine mapping and pyramiding of brown planthopper resistance genes QBph3 and QBph4 in an introgression line from wild rice O. officinalis. Mol Breed. 2015;35(1):1-10.

8. Du B, Zhang W, Liu B, Hu J, Wei Z, Shi Z, et al. Identification and characterization of Bph14, a gene conferring resistance to brown plant hopper in rice. Proc Natl Acad Sci U S A. 2009;106(52):22163-8.

9. Liu Y, Wu H, Chen H, Liu Y, He J, Kang H, et al. A gene cluster encoding lectin receptor kinases confers broad-spectrum and durable insect resistance in rice. Nat Biotechnol. 2015;33(3):301-5.

10. Tamura Y, Hattori M, Yoshioka H, Yoshioka M, Takahashi A, Wu JZ, et al. Map-based cloning and characterization of a brown planthopper resistance gene BPH26 from Oryza sativa L. ssp indica cultivar ADR52. Sci Rep. 2014:4:5872

11. Wang Y, Cao L, Zhang Y, Cao C, Liu F, Huang F, et al. Map-based cloning and characterization of $\mathrm{BPH} 29$, a $\mathrm{B} 3$ domain-containing recessive gene conferring brown planthopper resistance in rice. J Exp Bot. 2015;66(19):6035-45.

12. Zhou G, Qi J, Ren N, Cheng J, Erb M, Mao B, et al. Silencing OsHI-LOX makes rice more susceptible to chewing herbivores, but enhances resistance to a phloem feeder. Plant J. 2009;60(4):638-48.

13. Hu J, Zhou J, Peng X, Xu H, Liu C, Du B, et al. The Bphi008a gene interacts with the ethylene pathway and transcriptionally regulates MAPK genes in the response of rice to brown planthopper feeding. Plant Physiol. 2011;156(2):856-72.

14. Lu J, Ju H, Zhou G, Zhu C, Erb M, Wang X, et al. An EAR-motif-containing ERF transcription factor affects herbivore-induced signaling, defense and resistance in rice. Plant J. 2011;68(4):583-96.

15. Qi J, Lou Y. The chloroplast-localized phospholipases D a4 and a5 regulate herbivore-induced direct and indirect defenses in rice. Plant Physiol. 2011;157(4):1987-99.

16. Tong X, Qi J, Zhu X, Mao B, Zeng L, Wang B, et al. The rice hydroperoxide lyase OsHPL3 functions in defense responses by modulating the oxylipin pathway. Plant J. 2012;71(5):763-75.

17. Lu J, Li J, Ju H, Liu X, Erb M, Xia W, et al. Contrasting effects of ethylene biosynthesis on induced plant resistance against a chewing and a piercingsucking herbivore in rice. Mol Plant. 2014;7(11):1670-82.

18. Guo H, Li H, Zhou S, Xue H, Miao X. Cis-12-oxo-phytodienoic acid stimulates rice defense response to a piercing-sucking insect. Mol Plant. 2014;7(11):1683-92.
19. Zhou G, Ren N, Qi J, Lu J, Xiang C, Ju H, et al. The 9-lipoxygenase Osr9-LOX1 interacts with the 13-lipoxygenase-mediated pathway to regulate resistance to chewing and piercing-sucking herbivores in rice. Physiol Plant. 2014;152(1):59-69.

20. Qi J, Li J, Han X, Li R, Wu J, Yu H, et al. Jasmonic acid carboxyl methyltransferase regulates development and herbivory-induced defense response in rice. J Integr Plant Biol. 2015;58(6):564-76.

21. Yang $L$, Zhang W. Genetic and biochemical mechanisms of rice resistance to planthopper. Plant Cell Rep. 2016;35(8):1559-72.

22. Jain M, Nijhawan A, Tyagi AK, Khurana JP. Validation of housekeeping genes as internal control for studying gene expression in rice by quantitative realtime PCR. Biochem Biophys Res Commun. 2006;345(2):646-51.

23. Wang $L$, Xie W, Chen Y, Tang W, Yang J, Ye R, et al. A dynamic gene expression atlas covering the entire life cycle of rice. Plant J. 2010;61(5):752-66.

24. Vandesompele J, Preter KD, Pattyn F, Poppe B, Roy NV, Paepe AD, et al. Accurate normalization of real-time quantitative RT-PCR data by geometric averaging of multiple internal control genes. Genome Biol. 2002;3(7):research0034.1-research0034.11.

25. Sun L, Su C, Wang C, Zhai H, Wan J. Mapping of a major resistance gene to the brown planthopper in the rice cultivar Rathu Heenati. Breed Sci. 2005:55(4):391-6.

26. Jairin J, Phengrat $K$, Teangdeerith S, Vanavichit A, Toojinda T. Mapping of a broad-spectrum brown planthopper resistance gene, Bph3, on rice chromosome 6. Mol Breed. 2007;19(1):35-44.

27. Jairin J, Teangdeerith S, Leelagud $P$, Phengrat $K$, Vanavichit A, Toojinda T. Detection of brown planthopper resistance genes from different rice mapping populations in the same genomic location. Sci Asia. 2007;33(3):347-52.

28. Wang Y, Li H, Yuan S, Hao Z, Guo H, Miao X. Microarray analysis of broadspectrum resistance derived from an indica cultivar Rathu Heenati. Planta. 2012;235(4):829-40.

29. Wang Y, Guo H, Li H, Zhang H, Miao X. Identification of transcription factors potential related to brown planthopper resistance in rice via microarray expression profiling. BMC Genomics. 2012;13(1):687.

30. Cheng X, Zhu L, He G. Towards understanding of molecular interactions between rice and the brown planthopper. Mol Plant. 2013;6(3):621-34.

31. Lv W, Du B, Shangguan X, Zhao Y, Pan Y, Zhu L, et al. BAC and RNA sequencing reveal the brown planthopper resistance gene BPH15 in a recombination cold spot that mediates a unique defense mechanism. BMC Genomics. 2014;15(1):674.

32. Wang F, Ning D, Chen Y, Dang C, Han NS, Liu YE, et al. Comparing gene expression profiles between $\mathrm{Bt}$ and non-Bt rice in response to brown planthopper infestation. Front Plant Sci. 2015;6(603):1181.

33. Zheng Q, Wang XJ. GOEAST: a web-based software toolkit for Gene Ontology enrichment analysis. Nucleic Acids Res. 2008;36(Web Server issue): W358-63.

\section{Submit your next manuscript to BioMed Central and we will help you at every step:}

- We accept pre-submission inquiries

- Our selector tool helps you to find the most relevant journal

- We provide round the clock customer support

- Convenient online submission

- Thorough peer review

- Inclusion in PubMed and all major indexing services

- Maximum visibility for your research

Submit your manuscript at www.biomedcentral.com/submit
) Biomed Central 